This item was submitted to Loughborough's Research Repository by the author.

Items in Figshare are protected by copyright, with all rights reserved, unless otherwise indicated.

\title{
Semi-classical theory of magnetic quantum oscillations in a two-dimensional multiband canonical Fermi liquid
}

PLEASE CITE THE PUBLISHED VERSION

LICENCE

CC BY-NC-ND 4.0

\section{REPOSITORY RECORD}

Alexandrov, A.S., and A.M. Bratkovsky. 2019. "Semi-classical Theory of Magnetic Quantum Oscillations in a Two-dimensional Multiband Canonical Fermi Liquid". figshare. https://hdl.handle.net/2134/1154. 


\title{
Semi-classical theory of magnetic quantum oscillations in a two-dimensional multiband canonical Fermi liquid.
}

\author{
A.S. Alexandrov ${ }^{1}$ and A.M. Bratkovsky ${ }^{2}$ \\ (1) Department of Physics, Loughborough University, LE11 3TU, United Kingdom \\ ${ }^{(2)}$ Hewlett-Packard Laboratories, 1501 Page Mill Road, Palo Alto, California 94304
}

(July 20, 2000)

\begin{abstract}
The semi-classical Lifshitz-Kosevich (LK) description of quantum oscillations is extended to a multiband two-dimensional Fermi liquid with a constant number of electrons. The amplitudes of novel oscillations with combination frequencies, recently predicted and observed experimentally, are analytically derived and compared with the single-band amplitudes. The combination amplitudes decay with temperature exponentially faster than the standard harmonics, and this provides a valuable tool for their experimental identification.
\end{abstract}

71.25.Hc, 71.18.+y, 71.10.Ay, 71.70.Di, 71.10.Pm

It has been shown [1] that the magnetic quantum osin cillations in a multiband $2 D$ metal with a fixed electron $\checkmark$ density (canonical ensemble $(C E)$ ) are qualitatively different from those in an open system where the chemi- cal potential is fixed (grand canonical ensemble $(G C E)$ ).

$>$ There is a mechanism for different bands to 'talk' to each 6 other in $C E$ producing a dHvA signal with the combina- tion frequencies, $f=f_{1}+f_{2}$ [1] and $f=f_{1}-f_{2}$ [2, 3] in addition to the ordinary $\mathrm{dHvA}$ frequencies, $f_{1,2}$, of the individual bands in GCE. Numerical studies of novel oscillations showed that their amplitudes are comparable with the standard components, and they are robust with respect to a background (non-quantized) density of states [2].

The novel frequencies have been recently observed (4) in quantum well structures. These additional components in the dHvA frequency spectrum of lowdimensional metals may provide a unique information on the Fermi surface and carrier density if detailed analytical theory is available. In this paper we develop such a theory in the framework of the semiclassical LK approach [5]. The equations (12) and (13) are the main results of the present paper.

We first derive a convenient expression for a multiband two-dimensional thermodynamic potential in an external magnetic field $H$,

$$
\Omega=-T \int d \epsilon N(\epsilon, B) \ln \left[1+\exp \left(\frac{\mu-\epsilon}{T}\right)\right]
$$

where

$$
N(\epsilon, B)=\sum_{\alpha} \sum_{n=0}^{\infty} \rho_{\alpha} \omega_{\alpha} \delta\left(\epsilon-\epsilon_{\alpha n}\right)
$$

is the quantized density of states, $\rho_{\alpha}$ is the zero-field density of states in the band $\alpha, \epsilon_{\alpha n}=\Delta_{\alpha 0}+\omega_{\alpha}(n+1 / 2)+$ $g_{\alpha} \sigma \mu_{B} B, \omega_{\alpha}=e B / m_{\alpha}$ the cyclotron frequency with the cyclotron mass $m_{\alpha}, B=H+4 \pi M$ the magnetic field,
$\Delta_{\alpha 0}$ the band edge in zero magnetic field, $\mu$ the chemical potential, $g_{\alpha}$ the electron $g$-factor, $\sigma= \pm 1 / 2, \mu_{B}$ the Bohr magneton, and $\hbar=c=k_{B}=1$. The band index $\alpha$ includes the electron spin. In actual experiments on 2DEG $B \approx H$ and magnetic coupling between subbands was negligible [4].

By applying the Poisson formula [6] to the sum over $n$ in the thermodynamic potential

$$
\Omega=-T \sum_{\alpha} \rho_{\alpha} \omega_{\alpha} \sum_{n=0}^{\infty} \ln \left[1+\exp \left(\frac{\mu_{\alpha}-\omega_{\alpha}(n+1 / 2)}{T}\right)\right]
$$

with $\mu_{\alpha}=\mu-\Delta_{\alpha}$ and $\Delta_{\alpha}=\Delta_{\alpha 0}+g_{\alpha} \sigma \mu_{B} B$, we obtain

$$
\Omega=\Omega_{0}+\tilde{\Omega},
$$

where

$$
\Omega_{0}=-T \int_{0}^{\infty} d \epsilon \sum_{\alpha} \rho_{\alpha} \ln \left[1+\exp \left(\frac{\mu_{\alpha}-\epsilon}{T}\right)\right]
$$

is the 'classical' part. In $G C E$ it does not oscillate as a function of $1 / B$, and contains the contribution due to spin susceptibility (Pauli paramagnetism). The second part is

$$
\tilde{\Omega}=\frac{1}{24} \sum_{\alpha} \rho_{\alpha} \omega_{\alpha}^{2}+2 \sum_{\alpha} \sum_{r=1}^{\infty} A_{\alpha}^{r} \cos \left(\frac{r f_{\alpha}}{B}+\phi_{\alpha}^{r}\right),
$$

where the first term produces the Landau diamagnetism and the second oscillatory term is responsible for the de Haas-van Alphen effect. It is small compared with the 'classical' part as $\tilde{\Omega} / \Omega_{0} \sim(\omega / \mu)^{2}$. The Fourier components appear with frequencies $r f_{\alpha} \equiv r S_{\alpha} / e$, where $S_{\alpha}=2 \pi m_{\alpha}\left(\mu-\Delta_{\alpha 0}\right)$ is the Fermi surface zero-field crosssection. The amplitudes of the Fourier harmonics are

$$
A_{\alpha}^{r}=\frac{T \rho_{\alpha} \omega_{\alpha}}{2 r \sinh \left(2 \pi^{2} r T / \omega_{\alpha}\right)}
$$


and the phase $\phi_{\alpha}^{r}=r \pi\left(1+g_{\alpha} \sigma\right)$.

Differently from $G C E$, the chemical potential oscillates in $C E$. Hence, the 'classical' part of $\Omega$ contributes to oscillations as well. The relevant thermodynamic potential of $C E$ is the free energy $F=\Omega+\mu N$, with a fixed number of electrons, $N=-\partial \Omega / \partial \mu$. At low temperatures we find

$$
\Omega_{0}=-\sum_{\alpha} \rho_{\alpha} \mu_{\alpha}^{2} / 2
$$

so that

$$
\mu=\frac{1}{\rho}\left(N+\sum_{\alpha} \rho_{\alpha} \Delta_{\alpha}+\frac{\partial \tilde{\Omega}}{\partial \mu}\right),
$$

where $\rho=\sum_{\alpha} \rho_{\alpha}$ is the total density of states. Substituting this expression into $\Omega_{0}$, Eq.(8), we obtain

$$
F=F_{0}+\tilde{F},
$$

where the smooth non-oscillatory part of the free energy is given by

$$
F_{0}=\frac{1}{2 \rho}\left(N+\sum_{\alpha} \rho_{\alpha} \Delta_{\alpha}\right)^{2}-\frac{1}{2} \sum_{\alpha} \rho_{\alpha} \Delta_{\alpha}^{2},
$$

while the most essential oscillatory part is

$$
\tilde{F}=\tilde{\Omega}-\frac{1}{2 \rho}\left(\frac{\partial \tilde{\Omega}}{\partial \mu}\right)^{2} .
$$

In a more explicit form we obtain

$$
\begin{aligned}
\tilde{F} & =\frac{1}{24} \sum_{\alpha} \rho_{\alpha} \omega_{\alpha}^{2}+2 \sum_{\alpha, r} A_{\alpha}^{r} \cos \left(\frac{r f_{\alpha}}{B}+\phi_{\alpha}^{r}\right) \\
& -4 \sum_{\alpha, \alpha^{\prime}, r, r^{\prime}} C_{\alpha \alpha^{\prime}}^{r r^{\prime}} \sin \left(\frac{r f_{\alpha}}{B}+\phi_{\alpha}^{r}\right) \sin \left(\frac{r^{\prime} f_{\alpha^{\prime}}}{B}+\phi_{\alpha^{\prime}}^{r^{\prime}}\right) .
\end{aligned}
$$

It is the last term, which yields combination Fourier harmonics with the combination frequencies $f=r f_{\alpha} \pm r^{\prime} f_{\alpha^{\prime}}$. Their amplitudes,

$$
C_{\alpha \alpha^{\prime}}^{r r^{\prime}}=2 \pi^{2} \frac{r r^{\prime} A_{\alpha}^{r} A_{\alpha^{\prime}}^{r^{\prime}}}{\rho \omega_{\alpha} \omega_{\alpha^{\prime}}}
$$

are comparable with the standard single-band harmonics at low temperatures, $T<\omega_{\alpha} / 2 \pi^{2} r$, as also found in the numerical analysis [1.2] and in the experiment [4]. For example, the ratio of the combination amplitude to a single-band one for $r=r^{\prime}=1$ and $T=0$ in a metal with two parabolic bands $\left(\rho_{\alpha}=m_{\alpha} / 2 \pi\right)$ is

$$
\frac{2 C_{\alpha \alpha^{\prime}}^{11}}{A_{\alpha}^{1}}=\frac{m_{\alpha}}{m_{\alpha}+m_{\alpha^{\prime}}} \text {. }
$$

Differentiating $\tilde{\Omega}$ in $G C E$ and $\tilde{F}$ in $C E$ with respect to $H$ one obtains the ratio of the combination and single band amplitudes of magnetization as

$$
\frac{M_{\alpha \alpha^{\prime}}^{11}}{M_{\alpha}^{1}}=\frac{2 C_{\alpha \alpha^{\prime}}^{11}}{A_{\alpha}^{1}} \frac{f}{f_{\alpha}}=\frac{m_{\alpha}}{m_{\alpha}+m_{\alpha^{\prime}}} \frac{f}{f_{\alpha}},
$$

Differentiating twice the ratio in susceptibility is $(T=0)$

$$
\frac{\chi_{\alpha \alpha^{\prime}}^{11}}{\chi_{\alpha}^{1}}=\frac{2 C_{\alpha \alpha^{\prime}}^{11}}{A_{\alpha}^{1}}\left(\frac{f}{f_{\alpha}}\right)^{2}=\frac{m_{\alpha}}{m_{\alpha}+m_{\alpha^{\prime}}}\left(\frac{f}{f_{\alpha}}\right)^{2} .
$$

Last two ratios may be even larger than unity for the 'plus' combination harmonic $\left(f=f_{\alpha^{\prime}}+f_{\alpha}\right)$ while the 'minus' one $\left(f=f_{\alpha^{\prime}}-f_{\alpha}\right)$ is suppressed in magnetization and susceptibility, Fig.1. At higher temperatures the combination harmonics are exponentially small compared with the single-band ones, as shown in Fig.1.

We note that according to Eq.(13) the difference between the second (and higher) Fourier amplitudes for open and closed systems should be seen even in a simplest single-band metal. On the other hand in three (and higher)- band metals a mixture of more than two different frequencies can be observed due to non-parabolic band dispersion giving rise to cubic and higher powers of the chemical potential in the expression for $\Omega_{0}$, Eq.(8). In very high magnetic fields the usual magnetic breakdown and the non-linear field dependence of magnetic subbands due to non-parabolicity of the band dispersion could also lead to combination frequencies [7].

In conclusion we have developed the analytical semiclassical theory of magnetic quantum oscillations in multi-band two-dimensional metals. We have found the amplitudes of the novel combination Fourier harmonics, which are comparable with the single-band harmonics at low temperatures and exponentially small at higher temperatures. Their frequencies and the temperature dependence of amplitudes provide additional information on the band structure and carrier densities of a multiband canonical Fermi liquid. Essentially different temperature dependence of the combination amplitudes compared with the standard harmonics, Fig.1, should allow to distinguish them experimentally.

We thank A.P. Levanyuk for enlightening comments on the relationship between different statistical ensembles.

[1] A.S. Alexandrov and A.M. Bratkovsky, Phys. Rev. Lett. 76, 1308 (1996).

[2] A.S. Alexandrov and A.M. Bratkovsky, Phys. Lett. A 234, 53 (1997).

[3] M. Nakano, J. Phys. Soc. Jpn. 6619 (1997).

[4] R.A. Shepherd, M. Elliott, W.G. Herrenden-Harker, M. Zervos, P.R. Morris, M. Beck and M. Ilegems, Phys. Rev. B60, R11277 (1999).

[5] I.M. Lifshitz and A.M. Kosevich, Zh. Eksp. Teor. Fiz., 29, 730 (1955). 


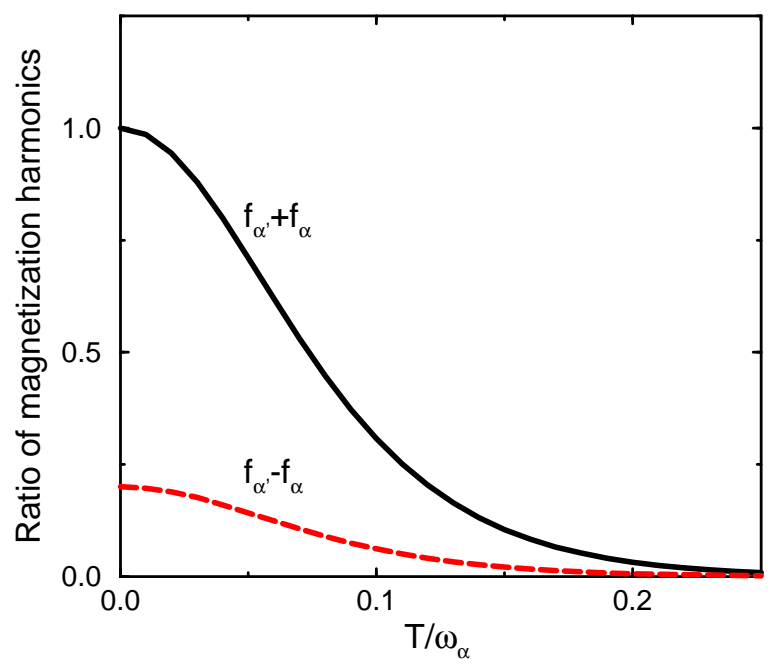

FIG. 1. The relative values of $f_{\alpha^{\prime}}+f_{\alpha}$ and $f_{\alpha^{\prime}}-f_{\alpha}$ combination Fourier components of magnetization for $m_{\alpha^{\prime}}=1.5 m_{\alpha}$ and $f_{\alpha^{\prime}}=1.5 f_{\alpha}$. The ratio for the parabolic bands is $M_{\alpha \alpha^{\prime}}^{11} / M_{\alpha}^{1}=2 \pi^{2} T m_{\alpha^{\prime}}\left(f_{\alpha^{\prime}} \pm f_{\alpha}\right) /$ $\left[f_{\alpha}\left(m_{\alpha}+m_{\alpha^{\prime}}\right) \omega_{\alpha} \sinh \left(2 \pi^{2} T / \omega_{\alpha^{\prime}}\right)\right]$.

[6] D. Shoenberg, Magnetic Oscillations (Cambridge Univ. Press, Cambridge, 1984), p. 481.

[7] S.Y. Han, J.S. Brooks, and J.H. Kim, Phys. Rev. Lett. 85, No.5 (2000). 